\title{
Hzf and hCAS/CSE1L: making the right choice in p53-mediated tumour suppression
}

\author{
Katherine E Ewings ${ }^{1}$, Kevin M Ryan ${ }^{1}$ \\ ${ }^{1}$ Tumour Cell Death Laboratory, Beatson Institute for Cancer Research, Cancer Research UK Beatson Laboratories, Garscube Estate, \\ SwitchbackRd, Glasgow, G611BD,UK.k.ryan@beatson.gla.ac.uk
}

Cell Research (2007) 17:829-831.doi: 10.1038/cr.2007.85; published online 15 October 2007

Preventing the propagation of damaged cells is a central component of tumour suppression. A key factor in this process is the transcription factor $\mathrm{p} 53$ - a fact exemplified by its frequent inactivation in human cancer. Since its discovery, over forty thousand reports have been published investigating $\mathrm{p} 53$ function and regulation. It is known that p53 mediates the expression of a diverse set of target genes which are broadly grouped by the biological response they provoke, with the best characterized being the induction of growth arrest, during which cellular damage is repaired, or the induction of apoptosis, which serves to eradicate damaged cells that may otherwise go on to form a tumour [1]. Despite the vast amount of work published, a central question remains: how does p53 initiate the appropriate biological programme in response to a specific stimulus? Clearly this is an important issue as either an unrestrained or inadequate response will have a fundamental impact upon the cell's, if not organism's, survival.

p53 is subject to multiple levels of regulation and different regulatory pathways are proposed to be responsible for guiding its biological outcome [1]. p53 undergoes many post-translational modifications including phosphorylation, ubiquitination and acetylation. These serve to not only regulate the stability of $\mathrm{p} 53$ but also affect its interactions with partner proteins and regulate target gene selection. The affinity of p53 for binding sites within target gene promoters is also known to be variable and it has been suggested that high affinity p53-binding sites associate with growth arrest related genes whilst low affinity sites cluster with pro-apoptotic genes. This hypothesis may help to explain the observation that low levels of p 53 can in some cases result in cell cycle arrest whereas higher levels of damage and p53 induce apoptosis. In a similar manner, the
DNA topology of p53 response elements may also serve as a structural determinant to influence promoter choice. Finally, p53 is known to interact with numerous co-factors to modulate target gene selection.

p53 binding proteins are known to influence p53 activity by various methods. A number of factors act by binding to $\mathrm{p} 53$ and modifying its target gene selection. The ASPP family members, ASPP1 and ASPP2 interact with p53's DNA binding domain and increase its transcriptional activity towards promoters of pro-apoptotic genes [2]; in contrast another family member iASPP interferes with the activation of pro-apoptotic genes [3]. Another example of p53 binding proteins whose family members have opposing actions is the Brn3 family of POU domain transcription factors. The direct interaction between Brn3a and p53 represses p53's ability to transactivate the pro-apoptotic Bax promoter whilst enhancing transcription of the cellcycle arrest gene p21 the outcome being cell cycle arrest, in comparison Brn3b promotes apoptosis [4]. In addition to these proteins which determine promoter binding, a number of other $\mathrm{p} 53$-binding proteins have been proposed to act on chromatin to alter target gene selection. The $\mathrm{CBP} / \mathrm{p} 300$ family of acetyltransferases bind directly to p53's activation domain and acetylate both p53 and histones, altering p 53 target gene selection. In addition, other factors including CARM1, PRMT and JMY are known to cooperate with $\mathrm{p} 300$ to activate specific promoters during a p53 response [5].

The importance of $\mathrm{p} 53$ co-factors in mediating cell fate decisions is illustrated by the observation that their expression is often deregulated in tumours, for example ASPP1 and 2 are frequently down-regulated whilst iASPP is overexpressed $[2,3]$. Consequently the identification of these 
proteins is critical in fully understanding p53 activation and influence on biological outcome. Two recent papers in Cell provide further insights into $\mathrm{p} 53$ regulation and describe the identification of two factors which modulate $\mathrm{p} 53$ 's target gene selection and biological response.

In a report by Das et al. hematopoietic zinc finger (Hzf), a p53 target gene, is identified as a modulator of p53 activity [6]. They propose that upon binding to Hzf, p53 is preferentially recruited to the promoters of cell-cycle arrest mediating genes. The authors demonstrate that $\mathrm{p} 53$ 's ability to transactivate specific target gene promoters is directly regulated by Hzf, since either its knockdown by RNA interference (RNAi) or gene ablation, compromises p53's ability to promote expression of cell-cycle arrest target genes whilst enhancing the transactivation of proapoptotic genes. Importantly they show that in the presence of Hzf, p53-mediated cell-cycle arrest is promoted but in its absence the apoptotic function of $\mathrm{p} 53$ is enhanced, crucially this can be suppressed by ectopic expression of Hzf.

As previously discussed it has been proposed that the level of damage inflicted upon a cell can serve as a guide for the response initiated by $\mathrm{p} 53$. This paper suggests that Hzf expression level may play a critical role in this decision. Through monitoring the expression of Hzf during DNA damage, the authors observed that following prolonged DNA damage both the total protein level and the amount of Hzf bound to p53 rapidly declined. Interestingly this decline correlated with the up-regulation of pro-apoptotic genes and induction of apoptosis. The inhibition of protein turnover, by proteasomal inhibition, was able to inhibit both the destabilization of Hzf and p53-mediated apoptosis, therefore raising the possibility that Hzf plays a critical role in the p53-dependent switch between cell cycle arrest and apoptosis (Figure 1). It is therefore important to determine how Hzf protein stability is regulated and also determine if ectopic expression of Hzf alone can rescue cells from apoptosis at the switch point identified.

It is as yet unclear how Hzf directs p53 target gene selection or if it actively or passively represses pro-apoptotic gene expression. Since Hzf is unable to bind DNA directly, alternative methods to explain its ability to modify p53's gene selection should be investigated. Mechanisms could include recruitment of binding proteins or alterations in p53 conformation allowing alternate promoter recognition. Since the authors demonstrate that Hzf binding to tumourderived p53 mutants is compromised, but not abolished, it would be interesting to determine if Hzf plays a role in their target gene selection. Furthermore it would be informative to establish the status and regulation of $\mathrm{Hzf}$ in tumour cells, especially those in which p53 is not mutated.

The second of the reports describes the identification of $\mathrm{hCAS} / \mathrm{CSE} 1 \mathrm{~L}$ as a long range chromatin modifier which potentially acts independently of an interaction with p53 [7]. Using a combination of chromatin immunoprecipitation (ChIP), size fractionation and mass spectrometry, Tanaka et al. found hCAS/CSE1L within p53-DNA crosslinked complexes. They were able to show that down-regulation of hCAS/CSE1L by RNAi significantly inhibited the activation of a particular subset of target genes. However, in contrast to Hzf, the genes affected did not clearly fall into classical pro-apoptotic or pro-arrest classes but interestingly knockdown of hCAS/CSE1L was still able to reduce the level of apoptosis in MCF7 cells in response to UVC radiation.

The interaction of hCAS/CSE1L with various $\mathrm{p} 53$ target gene promoters does not require p53 binding to the promoter and similarly $\mathrm{p} 53$ does not require hCAS/CSE1L for promoter binding. Using the pro-apoptotic PIG3 promoter as an example, the authors demonstrated that the proteins interacted independently with different regions of the promoter. Therefore although the two proteins can be co-immunoprecipitated, there is no evidence for a direct

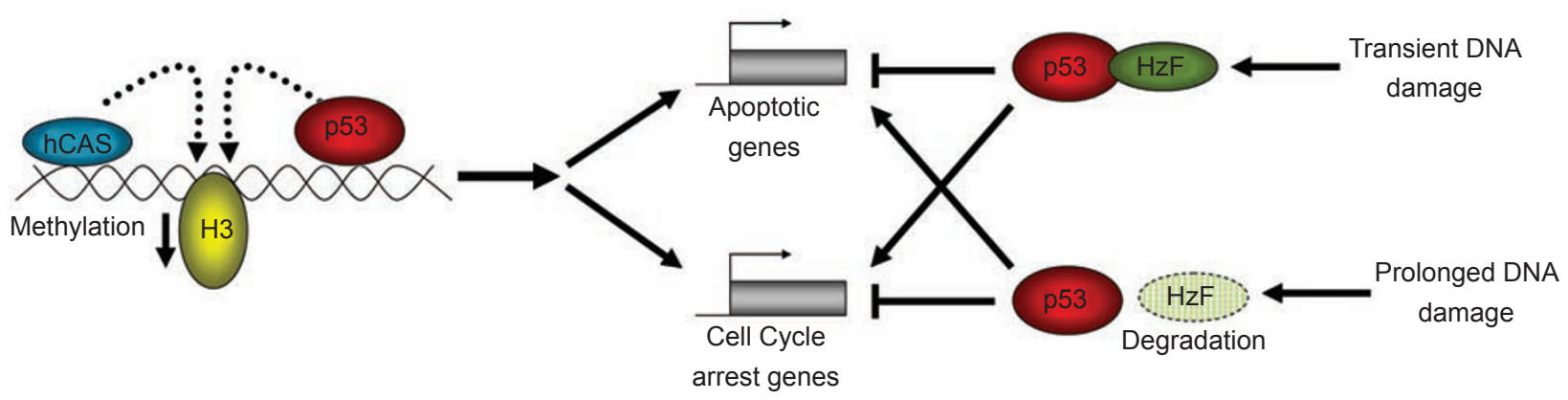

Figure 1 Regulation of p53 target gene selection. Under transient DNA damage Hzf binds to p53 and modulates it target gene selection promoting the expression of cell cycle arrest genes, following prolonged damage Hzf is degraded and p53 target gene selection switches to pro-apoptotic genes. In contrast, hCAS/CSE1L interacts with the promoters of p53 target genes and together with p53 downregulates the inhibitory lysine 27 methylation on histone H3, thereby modulating gene expression. 
interaction at $\mathrm{p} 53$ target gene promoters and so on a technical note, the method employed in this study is potentially a powerful tool in allowing the identification of functional, if not necessarily physical, interactions between proteins.

Using a combination of ChIP assays and RNAi it was shown that hCAS/CSE1L and p53 act together to downregulate methylation of lysine 27 on histone H3 (Figure 1). This modification is enriched in inactive heterochromatin and hence the authors propose that hCAS/CSE1L and p53 functionally interact to facilitate the formation of active chromatin at select targets genes. Exactly how they downregulate histone methylation is unclear and requires further investigation, as does the mechanism by which hCAS/ CSE1L binds to specific promoters. Since its localisation is independent of $\mathrm{p} 53$ it suggests that specific recognition motifs or alternate accessory proteins are required for the interaction and it would be informative to identify any such factors as these may potentially play a role in p53 target gene selection. Furthermore, since hCAS/CSE1L is known to be a component of the nuclear pore complex (NPC) and recent reports using yeast as a model system have suggested that the association of genes with the NPC is implicated in transcriptional regulation [8], this work also raises the intriguing possibility that sub-nuclear localisation may play a role in $\mathrm{p} 53$ target gene selection and regulation.

Although these two studies provide further insights into the regulation of $\mathrm{p} 53$ activity, many questions still remain. Since there are multiple $\mathrm{p} 53$ binding proteins, understanding the interplay between them is critical in determining how they act to modulate p53 activity. For example, do specific stress stimuli activate a particular co-factor or co-factors, or do specific co-factors display cell and tissue selective expression patterns? Also, do known p53 post-translational modifications alter these interactions? It would be interesting to know too whether these co-factors also regulate target genes involved in other p53-regulated processes such as the regulation of glycolysis or autophagy $[9,10]$. Answers to these questions will allow us to gain a fuller understanding of the complexity of p53 regulation and how it mediates cell fate decisions. Ultimately, it is hoped the knowledge gained will help design strategies to target the p53 pathway for therapeutic gain.

\section{References}

1 Crighton D, Ryan KM. Splicing DNA-damage responses to tumour cell death. Biochim Biophys Acta 2004; 1705:3-15.

2 Samuels-Lev Y, O'Connor DJ, Bergamaschi D, et al. ASPP proteins specifically stimulate the apoptotic function of $\mathrm{p} 53$. Mol Cell 2001; 8:781-794.

3 Bergamaschi D, Samuels Y, O’Neil NJ, et al. iASPP oncoprotein is a key inhibitor of p53 conserved from worm to human. Nat Genet 2003; 33:162-167.

4 Budhram-Mahadeo VS, Bowen S, Lee S, et al. Brn-3b enhances the pro-apoptotic effects of p53 but not its induction of cell cycle arrest by cooperating in trans-activation of bax expression. Nucleic Acids Res 2006; 34:6640-6652.

5 Coutts AS, La Thangue NB. The p53 response: emerging levels of co-factor complexity. Biochem Biophys Res Commun 2005; 331:778-785.

6 Das S, Raj L, Zhao B, et al. Hzf Determines Cell Survival upon Genotoxic Stress by Modulating p53 Transactivation. Cell 2007; 130:624-637.

7 Tanaka T, Ohkubo S, Tatsuno I, Prives C. hCAS/CSE1L Associates with Chromatin and Regulates Expression of Select p53 Target Genes. Cell 2007; 130:638-650.

8 Casolari JM, Brown CR, Komili S, West J, Hieronymus H, Silver PA. Genome-wide localization of the nuclear transport machinery couples transcriptional status and nuclear organization. Cell 2004; 117:427-439.

9 Bensaad K, Tsuruta A, Selak MA, et al. TIGAR, a p53-inducible regulator of glycolysis and apoptosis. Cell 2006; 126:107-120.

10 Crighton D, Wilkinson S, O'Prey J, et al. DRAM, a p53-induced modulator of autophagy, is critical for apoptosis. Cell 2006; 126:121-134. 